\title{
Gamma discounters are short-termist
}

\author{
Christian Gollier ${ }^{1}$ \\ Toulouse School of Economics
}

October 2014

\begin{abstract}
Weitzman (1998, 2001) proposed a simple "gamma discounting” method to characterize the term structure of discount rates today from the sole distribution of future spot interest rates. This rule which justifies using a smaller discount rate for longer maturities is now used for long-term policy evaluations in the UK, France, Norway, and potentially in the US. But we show that there is no social preference within the discounted expected utility framework that generically supports this pricing model and its underlying criterion, the expected net present value rule. Considering a standard Lucas tree economy, we characterize the term structure from a coherent joint distribution of future spot interest rates and future consumption levels. When future growth rates are positively serially correlated, efficient discount rates today are decreasing with maturity, and the gamma discounting rule yields discount rates that are larger than the efficient ones.
\end{abstract}

Keywords: decreasing discount rates, term structure, uncertain growth, Weitzman-Gollier puzzle.

JEL Codes: G11, G12, E43, Q54.

\footnotetext{
${ }^{1}$ The first version of this paper was entitled "An economic justification of gamma discounting”. I thank Michel Denuit, Mark Freeman, Terrence Iverson and Martin Weitzman for helpful discussions on this topic. The research leading to these results has received funding from the Chairs "Risk Markets and Value Creation" (SCOR) and "Sustainable Finance and Responsible Investments" at TSE, and from the European Research Council under the European Community's Seventh Framework Programme (FP7/2007-2013) Grant Agreement no. 230589.
} 


\section{Introduction}

The exponential nature of discounting at almost any reasonable positive discount rate implies that, when comparing alternative investments, their long term impacts do not really matter. This so-called short-termism intrinsic to standard discounting has been much criticized, in particular in the context of climate change. However, economic theory does not constrain discount factors to be exponential, or discount rates to be constant. ${ }^{2}$ Over the last 10 years or so, the United Kingdom (HM Treasury (2003)), France (Lebègue (2005)) and Norway (Official Norwegian Report (2012)) have used decreasing discount rates for the evaluation of public policies (in particular to estimate the social cost of carbon). In 2006, the OECD has published a cost-benefit manual (Pearce, Atkinson and Mourato, (2006)) that endorses this method. Moreover, the U.S. could consider a revision of the long-term discount rate by allowing it to be smaller than the short-term one (Arrow et alii (2013, 2014)). Decreasing discount rates introduce a potentially crucial bias in favor of investments that yield safe socioeconomic benefits in the distant future. In the U.K. for example, the term structure of discount rates goes from $3.5 \%$ for the short term, to $1 \%$ for long maturities. ${ }^{3}$ Thus, a shortterm policy with a safe return of $3 \%$ is dominated by a long-term project with a safe return of only $1 \%$.

Weitzman (1998, 2001) provided a simple argument that played a key role in the change of the evaluation rules prevailing in these countries. Consider a risk free project with a $\operatorname{cost} C$ to be paid today, and a single sure benefit $F$ occurring in $t$ years. If the compounded interest rate for this maturity is $r$, the future benefit of the project can be transferred to the present by a loan of $F \exp (-r t)$ today to be reimbursed at the termination date $t$. The portfolio containing the project and the loan has a single payoff which occurs today and is equal to the project's Net Present Value $N P V=-C+F \exp (-r t)$. Obviously, as long as one prefers more to less, this project is desirable if and only if its NPV is positive. Following Weitzman, suppose alternatively that $r$ is uncertain, so that the NPV of the safe project is uncertain. In that context, it is intuitive that a risk-neutral investor should invest in the project only if its expected NPV is positive. This expected NPV approach implies that, before observing $r$, one

\footnotetext{
${ }^{2}$ There is a normative argument based on the time consistency of decisions in favor of using a constant rate of pure preference for the present to discount future changes in utility in the intertemporal social welfare function. In this paper, we refer to the discount rate as the rate at which future changes in consumption are discounted. As long as its (potentially decreasing) term structure is derived from the exponentially discounted expected utility model, it is time consistent.

${ }^{3}$ In France, it goes from $4 \%$ to $2 \%$.
} 
should use the certainty equivalent rate of return of capital $r_{0 \rightarrow t}^{W}$ to discount a sure cash flow of maturity $t$, with $\exp \left(-r_{0 \rightarrow t}^{W} t\right)=E \exp (-r t)$. This discount rate is decreasing in maturity $t$, and it tends asymptotically to the smallest plausible interest rate. This argument for a decreasing term structure of the discount rate was first presented by Weitzman $(1998,2001)$, followed by Newell and Pizer (2003), Hepburn and Groom (2007), Groom, Koundouri, Panopoulou and Pantelidis (2007), Gollier, Koundouri and Pantelidis (2008), Freeman (2010), Freeman and Groom (2010), Traeger (2013), Arrow et alii (2013, 2014), and Farmer, Geanakoplos, Masoliver, Montero and Perello (2014) for example. Because Weitzman (2001) used a gamma distribution for $r$, this approach based on the expected NPV is often referred to as "gamma discounting”.

In this paper, we explore the economic foundation of gamma discounting. Various authors criticized the use of the expected NPV criterion to value safe projects when the discount rate is uncertain. We review this literature in Section 5 of this paper. Following Pazner and Razin (1975) and Gollier (2004), one could alternatively bring the net benefit of the project forward to the terminal date $t$ by a loan of $C$ today. This generates a net increase in consumption by $N F V=F-C \exp (r t)$ at the termination date $t$. Under an uncertain interest rate $r$, the riskneutral investor would find the project desirable if and only if its expected NFV is positive, yielding the certainty equivalent discount rates $r_{0 \rightarrow t}^{G}$ with $\exp \left(r_{0 \rightarrow t}^{G} t\right)=E \exp (r t)$. Observe that the discount rate $r_{0 \rightarrow t}^{G}$ is increasing and tends asymptotically to the largest possible $r$, thereby justifying a shape of the term structure exactly opposite to gamma discounting. This is the socalled Weitzman-Gollier paradox.

This paradox is related to the analysis initiated by Cox, Ingersoll and Ross (1981) who demonstrated that the different versions of the traditional "Expectations Hypothesis" (EH) used since Macaulay (1938) to price bonds are all incompatible to each other. For example, under one interpretation of the $\mathrm{EH}$, the conditional expected rates of return on bonds of all maturities over the next period are assumed to be equal to each other. It is easy to check that this version of the EH is equivalent to the expected NPV rule. Another interpretation of the EH equates the expected return of the buy-and-hold strategy of a long bond and the expected return of the strategy to roll-over short term bonds. It is immediate that this interpretation of the $\mathrm{EH}$ is equivalent to the expected NFV rule. In other words, the so-called WeitzmanGollier paradox is just a new incarnation of the old debate on the expectations hypothesis. The 
many different versions of the $\mathrm{EH}$ are all based on the idea that the representative agent is risk neutral. They are incompatible to each other and are all widely rejected by the data. ${ }^{4}$

In order to solve the puzzle, observe first that in the standard discounted expected utility model with a representative agent, the risk-neutrality assumption underlying the two discounting rules is technically incompatible with an uncertain interest (or discount) rate. Indeed, the Ramsey rule (Ramsey (1928)) tells us that if the representative agent is riskneutral, the interest rate should be equal to the rate of pure preference for the present of the representative agent, which is certain. Thus, in order to reconcile the basic ingredient of the gamma discounting approach, i.e. uncertain interest rates, with economic theory, one needs to consider a model with a risk-averse representative agent. This is the objective of this paper in which we consider the classical Lucas tree economy with an uncertain growth rate of consumption and a risk-averse representative agent. We characterize simultaneously the distribution of future spot interest rates and the term structure of efficient discount rates today, and we compare it to the term structure of gamma discount rates.

The incompatibility between consumption-based pricing models and the Expected NPV rule raises the question of the sign and the intensity of the error generated by users of this rule. As a preview of the main results of the paper, suppose that shocks to the growth rate of consumption are persistent, as documented for example by Bansal and Yaron (2004). This implies that future consumption is positively correlated to future spot interest rates. This implies in turn that the present value evaluated at $t-1$ of a sure benefit occurring at $t$ is negatively correlated with consumption at $t-1$. In other words, transferring to the present a future sure benefit through a sequence of short-term loans has a negative consumption-based CAPM beta. The gamma discounting rule ignores this fact by implicitly assuming that this strategy has a zero beta. To illustrate, consider the case of a safe benefit $F$ occurring in 60 years. We want to price this asset (a zero-coupon bond) by using the 30-year interest rate observed today, together with the uncertain 30-year interest rate that will prevail in 30 years. Suppose that one plans to borrow in 30 years the present value of $F$ using the 30-year interest rate that will be observable at that time. Let us denote it $P V_{30}$. Seen from today, this value is uncertain, but one can measure the certainty equivalent of $P V_{30}$. Discounting this certainty equivalent benefit at the currently prevailing 30-year interest rate yields the social value of the

\footnotetext{
${ }^{4}$ See for example Froot (1989): "If the attractiveness of an economic hypothesis is measured by the number of papers which statistically reject it, the expectations theory of the term structure is a knockout.”
} 
asset today. The problem is that $P V_{30}$ will be negatively correlated with $C_{30}$, the consumption level in 30 years, if shocks to the economy are persistent. The self-insurance feature of $P V_{30}$ implies that its certainty equivalent is larger than its expectation. Thus, the expected NPV rule underestimates the true value of the long-dated benefit $F$. The gamma discount rate $r_{0 \rightarrow 60}^{W}$ is thus too large, yielding an evaluation error that is qualitatively equivalent to discounting at the risk-free rate a cash flow with a negative CCAPM beta. Gamma discounting yields shorttermism.

In Section 2, we derive the standard consumption-based pricing formula for the term structure of efficient discount rates, and we show how this formula can be rewritten in the spirit of the expected NPV and expected NFV approaches. Section 3 is devoted to the comparison of these efficient rates and those derived from the gamma discounting rule. We also provide sufficient conditions for the efficient term structure to be increasing or decreasing. These results are derived for the two-period case. In Section 4, we generalize our analysis to the multi-period case.

\section{Efficient discount rates in a Lucas tree economy}

We consider a Lucas (1978) tree economy without any storage technology. The current crop and consumption by the representative agent is denoted $c_{0}$, and the beliefs about the evolution of future crops and consumption levels are given by the exogenous stochastic process $\left.c_{t}\right|_{t \geq 0}{ }^{5}$ We suppose that the representative agent evaluates her intertemporal welfare by computing the discounted flow of her expected utility, where $u$ represents her von Neumann-Morgenstern concave utility function. For the sake of simplicity, we assume that the rate of pure preference for the present is zero. ${ }^{6}$

We first characterize the discount rate that should be used at date $\tau \geq 0$ to evaluate a sure benefit occurring at date $t>\tau$, conditional on the information available at date $\tau$, which

\footnotetext{
${ }^{5}$ Our results are compatible with any general equilibrium model (as the simple AK model) that would make this stochastic consumption flow endogenous. Making the consumption process endogenous does not bring any new insight to the pricing problem.

${ }^{6}$ If the rate of pure preference for the present is not zero, all interest rates discussed in this paper should simply be reinterpreted as net of this rate.
} 
contains $c_{\tau}$. Obviously, at date $\tau$, the representative agent is just indifferent to invest in a project yielding $\Delta c_{\tau}=-\varepsilon$ and $\Delta c_{t}=\varepsilon \exp (t-\tau) r_{\tau \rightarrow t}$ if and only if

$$
u^{\prime}\left(c_{\tau}\right)=e^{(t-\tau) r_{\tau \rightarrow t}} E_{\tau} u^{\prime}\left(c_{t}\right)
$$

where $E_{\tau}$ is the expectation operator conditional on the information available at date $\tau$, with $E_{0}=E$. Equation (1) yields the efficient rate $r_{\tau \rightarrow t}$ to discount at date $\tau$ a safe benefit occurring at date $t$. In this section, we are particularly interested in the first two periods, for which equation (1) yields

$$
e^{-r_{0 \rightarrow 1}}=\frac{E u^{\prime}\left(c_{1}\right)}{u^{\prime}\left(c_{0}\right)}, \quad e^{-r_{1 \rightarrow 2}}=\frac{E_{1} u^{\prime}\left(c_{2}\right)}{u^{\prime}\left(c_{1}\right)},
$$

and

$$
e^{-2 r_{0 \rightarrow 2}}=\frac{E u^{\prime}\left(c_{2}\right)}{u^{\prime}\left(c_{0}\right)} .
$$

The spot short rate $r_{0 \rightarrow 1}$ and the spot long rate $r_{0 \rightarrow 2}$ are observable rates that characterize the term structure at date 0 . These are the efficient rates to be used at date 0 to discount safe benefits occurring at dates 1 and 2, respectively. They are also the equilibrium interest rates if markets are competitive and frictionless. At date 0 , the future spot interest rate $r_{1 \rightarrow 2}$ is in general uncertain since it depends upon information only available at date 1. Equations (2) and (3) are standard consumption-based pricing formulas. Following Weitzman (1998, 2001), we are interested in determining whether the long discount rate $r_{0 \rightarrow 2}$ can be expressed as a function of the spot rates $r_{0 \rightarrow 1}$ and $r_{1 \rightarrow 2}$ alone.

A simple way to compare an immediate benefit $\Delta c_{0}$ to a future benefit $\Delta c_{2}$ occurring in two periods is to invest $\Delta c_{0}$ in the safe asset yielding a sure return $r_{0 \rightarrow 1}$ in the first period, and then an uncertain return $r_{1 \rightarrow 2}$ in the second period. Because of the reinvestment risk, the difficulty of this approach is that it requires us to compare the safe future benefit $\Delta c_{2}$ to an uncertain future value $F V_{2}=\Delta c_{0} \exp \left(r_{0 \rightarrow 1}+r_{1 \rightarrow 2}\right)$ of $\Delta c_{0}$ using compounded interest rates. Obviously, they have the same impact on welfare if and only if

$$
\Delta c_{2} E u^{\prime}\left(c_{2}\right)=\Delta c_{0} E u^{\prime}\left(c_{2}\right) e^{r_{0 \rightarrow 1}+r_{1 \rightarrow 2}} .
$$


In other words, this "future valuation" approach yields a long discount rate $r_{0 \rightarrow 2}$ which is such that

$$
e^{2 r_{0 \rightarrow 2}}=E\left[\frac{u^{\prime}\left(c_{2}\right)}{E u^{\prime}\left(c_{2}\right)} e^{r_{0 \rightarrow 1}+r_{1 \rightarrow 2}}\right] .
$$

Consider alternatively the comparison of $\Delta c_{0}$ to the present value of $\Delta c_{2}$. Using $P V_{0}=\Delta c_{2} \exp \left(-r_{0 \rightarrow 1}-r_{1 \rightarrow 2}\right)$ for this purpose is problematic because the future spot rate $r_{1 \rightarrow 2}$ is generally unknown at date 0 . In other words, $P V_{0}$ does not correspond to any economically meaningful value, i.e., to an amount that could be borrowed today against the promise of a reimbursement $\Delta c_{2}$ in the future.

We can alternatively transfer the two benefits $\Delta c_{0}$ and $\Delta c_{2}$ to a welfare-equivalent increase consumption at date 1 . Benefit $\Delta c_{0}$ can be safely transformed into an increase in $c_{1}$ by $\Delta c_{0} \exp \left(r_{0 \rightarrow 1}\right)$. At date 1 , after observing the spot rate prevailing at that date, benefit $\Delta c_{2}$ can also be safely transformed into an immediate increase in consumption by $\Delta c_{2} \exp \left(-r_{1 \rightarrow 2}\right)$. However, when evaluated from date 0 , this payoff is uncertain. At date 0 , the two benefits have the same impact on intertemporal welfare if and only if

$$
\Delta c_{0} e^{r_{0 \rightarrow 1}}=\Delta c_{2} E\left[\frac{u^{\prime}\left(c_{1}\right)}{E u^{\prime}\left(c_{1}\right)} e^{-r_{1 \rightarrow 2}}\right]
$$

Notice that the right-hand side of this equality is the certainty equivalent of the present value of $\Delta c_{2}$ at date 1 . This equation is equivalent to discounting $\Delta c_{2}$ today at rate $r_{0 \rightarrow 2}$ such that

$$
e^{-2 r_{0 \rightarrow 2}}=E\left[\frac{u^{\prime}\left(c_{1}\right)}{E u^{\prime}\left(c_{1}\right)} e^{-r_{0 \rightarrow 1}-r_{1 \rightarrow 2}}\right] .
$$

To sum up, equations (3), (5) and (7) are three alternative ways to represent the same efficient long discount rate $r_{0 \rightarrow 2}$. Using condition (2), it is easy to check that they are equivalent. This is summarized in Proposition 1.

Proposition 1: There are three equivalent ways to define the efficient long discount rate $r_{0 \rightarrow 2}$ : 


$$
e^{-2 r_{0 \rightarrow 2}}=\frac{E u^{\prime}\left(c_{2}\right)}{u^{\prime}\left(c_{0}\right)}=\left(E\left[\frac{u^{\prime}\left(c_{2}\right)}{E u^{\prime}\left(c_{2}\right)} e^{r_{0 \rightarrow 1}+r_{1 \rightarrow 2}}\right]\right)^{-1}=E\left[\frac{u^{\prime}\left(c_{1}\right)}{E u^{\prime}\left(c_{1}\right)} e^{-r_{0 \rightarrow 1}-r_{1 \rightarrow 2}}\right] .
$$

Although equations (5) and (7) link the long discount rate today to the future spot interest rate, they fail to attain the objective envisioned by Weitzman $(1998,2001)$ to fully characterize the price of long-dated safe assets from the distribution of the sequence of spot interest rates alone. Indeed, the long discount rate described by equation (5) requires information about the distribution of consumption $c_{2}$ and its statistical relation to interest rates, whereas the long discount rate described by equation (7) requires information about the distribution of consumption $c_{1}$. Proposition 1 demonstrated that, under uncertainty, it is not possible to derive the efficient long discounting rule from the sole distribution of future spot rates.

\section{Link with gamma discounting and decreasing discount rates}

Weitzman (1998, 2001) proposed an alternative discount rate $r_{0 \rightarrow 2}^{W}$ which is based on the assumption that when future spot interest rates are uncertain, the efficient discount factor should be equal to the expected discount factor. This expected NPV rule yields

$$
\exp \left(-2 r_{0 \rightarrow 2}^{W}\right)=E \exp \left(-r_{0 \rightarrow 1}-r_{1 \rightarrow 2}\right)
$$

Notice that risk neutrality provides an inconsistent way to justify this "gamma discounting" rule from Proposition 1. When the representative agent is risk-neutral, it is right to say that conditions (7) and (9) yield the same discount rate $r_{0 \rightarrow 2}=r_{0 \rightarrow 2}^{W}$. But risk neutrality also implies from (2) that $r_{0 \rightarrow 1}=r_{1 \rightarrow 2}=0$ with certainty, so that the gamma discounting rule is trivially correct.

An alternative solution to match $r_{0 \rightarrow 2}^{W}$ with $r_{0 \rightarrow 2}$ is obtained by assuming that $c_{1}$ is known at date $0 .{ }^{7}$ In that case, by the Arrow-Lind Theorem, ${ }^{8}$ the representative agent is neutral to the marginal risk affecting the present value at date 1 of the sure benefit at date 2 .

\footnotetext{
${ }^{7}$ In the wording of Cox, Ingersoll and Ross (1981) and Gilles and Leroy (1986), this means that the dynamics of consumption are locally certain. These authors show that the price of long bonds has no risk or term premium under this assumption, so that the expected NPV rule is indeed efficient in that case.

${ }^{8}$ Arrow and Lind (1970) demonstrated that when there is no systematic risk, all marginal projects should be evaluated in a risk-neutral way.
} 
A third special case arises when future consumption $c_{1}$ is uncertain but statistically independent of the future spot interest rate $r_{1 \rightarrow 2}$. Equation (7) also implies that $r_{0 \rightarrow 2}=r_{0 \rightarrow 2}^{W}$ in that case. However, it has long been recognized that interest rates are statistically linked to the expected growth of consumption, and that consumption growth is itself subject to cycles. This implies that $c_{1}$ and $r_{1 \rightarrow 2}$ are generally not independent, so that $r_{0 \rightarrow 2}^{W} \neq r_{0 \rightarrow 2}$. To examine this problem further, we use the concept of Positive Quadrant Dependent (PQD), as defined by Lehmann (1966).

Definition: We say that a pair of random variables $\left(y_{1}, y_{2}\right)$ is PQD if for all $\left(a_{1}, a_{2}\right) \in \mathbb{R}^{2}$,

$$
\operatorname{Pr}\left[y_{1} \leq a_{1} \cap y_{2} \leq a_{2}\right] \geq \operatorname{Pr}\left[y_{1} \leq a_{1}\right] \operatorname{Pr}\left[y_{2} \leq a_{2}\right]
$$

This states that two random variables are PQD if the probability that they are simultaneously small is at least as great as when assuming that they are independent. In short, smaller values of $y_{1}$ go statistically with smaller values of $y_{2}$. PQD implies a positive correlation, but the converse statement is not true. However, in the special case of a bivariate normal distribution, PQD is equivalent to a positive correlation. In the economic literature, Levy and Paroush (1974) have been the first to show that when function $f: \mathbb{R}^{2} \rightarrow \mathbb{R}$ is supermodular, i.e., has a nonnegative cross derivative, then the PQD of $\left(x_{1}, x_{2}\right)$ implies that $E f\left(x_{1}, x_{2}\right)$ is larger than when assuming independence. ${ }^{9}$ The following proposition is a direct application of this result.

Proposition 2: Suppose that the future consumption level $c_{1}$ and the future spot rate $r_{1 \rightarrow 2}$ are positive quadrant dependent. Then, the gamma discount rate $r_{0 \rightarrow 2}^{W}$ defined by equation (9) is larger than the efficient discount rate $r_{0 \rightarrow 2}$.

Proof: Observe that function $f(c, r)=u^{\prime}(c) \exp (-r)$ is supermodular. If $c_{1}$ and $r_{1 \rightarrow 2}$ are PQD, equation (7) implies that

$$
\begin{aligned}
e^{-2 r_{0 \rightarrow 2}} & =\frac{e^{-r_{0 \rightarrow 1}}}{E u^{\prime}\left(c_{1}\right)} E\left[u^{\prime}\left(c_{1}\right) e^{-r_{1 \rightarrow 2}}\right] \geq \frac{e^{-r_{0 \rightarrow 1}}}{E u^{\prime}\left(c_{1}\right)} E\left[u^{\prime}\left(c_{1}\right)\right] E\left[e^{-r_{1 \rightarrow 2}}\right] \\
& =E\left[e^{-r_{0 \rightarrow 1}-r_{1 \rightarrow 2}}\right]=e^{-2 r_{0 \rightarrow 2}^{W}} .
\end{aligned}
$$

\footnotetext{
${ }^{9}$ Meyer and Strulovici (2012) provide a nice survey of the literature on PQD and its alternative definitions and terminologies, together with extensions from bivariate to multivariate functions.
} 
It implies that $r_{0 \rightarrow 2}^{W}$ is larger than $r_{0 \rightarrow 2} \cdot \square$

When shocks to the economic growth rate are persistent, they affect positively at the same time future consumption, and the future spot rate. If this positive correlation is strong enough to imply PQD, this proposition tells us that the discount rate $r_{0 \rightarrow 2}^{W}$ proposed by Weitzman overestimates the efficient discount rate $r_{0 \rightarrow 2}$. This is good news for the proponents of a decreasing term structure of discount rates who used the gamma discounting argument. The intuition of this result is simple. When consumption is positively correlated to interest rates, transferring a future safe benefit to the present by a loan yields a present value that is negatively correlated to present consumption. Ex ante, before observing consumption, this loan has the additional benefit to partially hedge the risk on consumption. This additional benefit is overlooked by gamma discounting, in which risk neutrality is assumed. Thus, the gamma discounting rule (9) underestimates the social benefit of distant cash flows, and yields long discount rates that are too large. The symmetric result holds when $c_{1}$ and $r_{1 \rightarrow 2}$ are negative quadrant dependent.

Proposition 2 also demonstrates that there is no social preference within the discounted expected utility framework that generically supports the gamma discounting rule of Weitzman (1998, 2001). In other words, for any utility function of the representative agent, one can find an economy $\left.c_{t}\right|_{t \geq 0}$ in which gamma discount rates are inefficient.

The result of Levy and Paroush (1974) can also be used to determine the slope of the term structure of efficient discount rates. Let $x_{t}=\ln c_{t} / c_{t-1}$ denote the growth rate of consumption during the time interval $[t-1, t]$. Observe then that the marginal utility at date 2 can be rewritten as $u^{\prime}\left(c_{0} \exp \left(x_{1}+x_{2}\right)\right)$, which is supermodular if and only if relative prudence $P(c)=-c u^{\prime \prime}(c) / u^{\prime \prime}(c)$ is uniformly larger than unity. This observation combined with equation (3) implies the following result, which is in Gollier (2014).

Proposition 3: Suppose that growth rates $\left(x_{1}, x_{2}\right)$ are positive quadrant dependent and that relative prudence is uniformly larger than unity. Then, the efficient long discount rate $r_{0 \rightarrow 2}$ is smaller than if serial growth rates would be independent with the same marginal distributions. 
The symmetric results hold when $\left(x_{1}, x_{2}\right)$ are negative quadrant dependent. But the recent literature on "long run risk" initiated by Bansal and Yaron (2004) tends to validate the hypothesis that growth rates of consumption are positively serially correlated.

Consider the special case with a CRRA utility function with $u^{\prime}(c)=c^{-\gamma}$ with $\gamma \geq 0$, so that relative prudence $P(c)=\gamma+1$ is uniformly larger than unity. The following corollary describes the two term structures in that case. It can be interpreted as a side result of Proposition 3, since the term structure of efficient discount rates is flat under CRRA when growth rates are serially i.i.d..

Corollary: Suppose that the representative agent has a CRRA utility function and that the serial growth rates of consumption $x_{1}$ and $x_{2}$ have the same marginal distribution. Then, the gamma discount rates have a flat term structure: $r_{0 \rightarrow 2}^{W}=r_{0 \rightarrow 1}$. Moreover, the term structure of efficient discount rates is decreasing $\left(r_{0 \rightarrow 2} \leq r_{0 \rightarrow 1}\right)$ if the pair of serial growth rates $\left(x_{1}, x_{2}\right)$ is positive quadrant dependent.

Proof: Because growth rates $x_{1}$ and $x_{2}$ have the same marginal distribution, we have that

$$
\begin{aligned}
e^{-2 r_{0 \rightarrow 2}^{W}} & =e^{-r_{0 \rightarrow 1}} E\left[e^{-r_{1 \rightarrow 2}}\right]=E e^{-\gamma x_{1}} E\left[E_{1} e^{-\gamma x_{2}}\right] \\
& =E e^{-\gamma x_{1}} E e^{-\gamma x_{2}}=\left(E e^{-\gamma x_{1}}\right)^{2}=e^{-2 r_{0 \rightarrow 1}} .
\end{aligned}
$$

This implies that $r_{0 \rightarrow 2}^{W}=r_{0 \rightarrow 1}$. Because function $f\left(x_{1}, x_{2}\right)=\exp -\gamma\left(x_{1}+x_{2}\right)$ is supermodular, we also have that

$$
e^{-2 r_{0 \rightarrow 2}}=E e^{-\gamma\left(x_{1}+x_{2}\right)} \geq\left(E e^{-\gamma x_{1}}\right)^{2}=e^{-2 r_{0 \rightarrow 1}}
$$

if $\left(x_{1}, x_{2}\right)$ is PQD. This concludes the proof of the Corollary.

Under the PQD of growth rates, the term structure of efficient discount rates is decreasing. Gollier (2012) illustrates the second part of this corollary by considering various stochastic processes for $c_{t}$ exhibiting different forms of positive serial interdependence. The link between prudence, positive serial interdependence of growth rates and the decreasing nature of the term structure of efficient rates is intuitive: the positive serial interdependence of growth rates magnifies the long term risk borne by the representative agent compared to the 
independent case in which the term structure is flat. This induces the prudent agent to bias investment decisions towards those that generate sure benefits in the more distant future. This is done by using decreasing discount rates. The first part of the Corollary shows that the gamma discounting rule fails to recognize this important aspect of choices over time. This failure is important enough to yield a flat term structure for $t=1,2$ for gamma discount rates under the assumptions of the corollary. This is illustrated by the following example.

Example 1: This example illustrates the findings of propositions 2 and 3, and of the corollary. We normalize $c_{0}=1$. We assume that $u^{\prime}(c)=c^{-\gamma}$ and that $\left(x_{1}, x_{2}\right)$ is joint normally distributed with $E x_{t}=\mu, \operatorname{Var}\left(x_{t}\right)=\sigma^{2}$ and $\operatorname{cov}\left(x_{1}, x_{2}\right)=\rho \sigma^{2}$. This means that $\left(x_{1}, x_{2}\right)$ is PQD if and only if $\rho$ is positive. We show in the Appendix that

$$
r_{1 \rightarrow 2} \mid c_{1}=\gamma \rho \ln c_{1}+\gamma(1-\rho) \mu-0.5 \gamma^{2} \sigma^{2}\left(1-\rho^{2}\right)
$$

This implies that $\left(c_{1}, r_{1 \rightarrow 2}\right)$ is jointly normal. The PQD assumption of $\left(c_{1}, r_{1 \rightarrow 2}\right)$ in Proposition 2 holds if and only if $\rho$ is positive. Finally, if $\rho$ is positive, we have that

$$
r_{0 \rightarrow 2}=\gamma \mu-0.5 \gamma^{2} \sigma^{2}(1+\rho) \leq \gamma \mu-0.5 \gamma^{2} \sigma^{2}=r_{0 \rightarrow 1}=r_{0 \rightarrow 2}^{W} .
$$

Observe that the efficient discount rate $r_{0 \rightarrow 2}$ is the sum of the risk free rate $\gamma \mu-0.5 \gamma^{2} \sigma^{2}$ and a risk premium $-0.5 \rho \gamma^{2} \sigma^{2}$. This risk premium equals half the product of the systematic risk premium $\gamma \sigma^{2}$ and the CCAPM beta of $P V_{1}=\exp \left(-r_{1 \rightarrow 2}\right)$. As seen from equation (14), this beta is constant and equal to $-\gamma \rho$.

\section{Generalization}

In this section, we generalize Proposition 1 to longer maturities. The main ingredients of this generalization are the spot rate $r_{\tau \rightarrow t}$ defined from equation (1) by

$$
e^{-(t-\tau) r_{\tau \rightarrow t}}=\frac{E_{\tau} u^{\prime}\left(c_{t}\right)}{u^{\prime}\left(c_{\tau}\right)},
$$

and the corresponding forward rate $R_{\tau \rightarrow t}$ defined by 


$$
e^{-(t-\tau) R_{\tau \rightarrow t}}=\frac{E u^{\prime}\left(c_{t}\right)}{E u^{\prime}\left(c_{\tau}\right)}=E\left[\frac{u^{\prime}\left(c_{\tau}\right)}{E u^{\prime}\left(c_{\tau}\right)} e^{-(t-\tau) r_{\tau \rightarrow t}}\right]
$$

This forward rate $R_{\tau \rightarrow t}$ is the rate to be used today to compare safe benefits occurring at dates $\tau$ and $t$. It is also the guaranteed interest rate that should be observed on forward credit contracts. Equation (17) tells us how to derive this forward rate from the joint distribution of the spot rate $r_{\tau \rightarrow t}$ and of consumption $c_{\tau}$. The interpretation of its right-hand side is based on the loan $P V_{\tau \leftarrow t}=\exp -(t-\tau) r_{\tau \rightarrow t}$ that can be obtained at date $\tau$ against a unit reimbursement at date $t>\tau$. Seen from date $0, P V_{\tau \leftarrow t}$ is uncertain. Of course, at the margin, the representative agent is indifferent at date 0 to consume 1 at date $t$, or the uncertain $P V_{\tau \leftarrow t}$ at date $\tau$. The RHS of (17) is the sure increase in consumption at date $\tau$ that has the same welfare effect at date 0 than consuming the uncertain payoff $P V_{\tau \leftarrow t}$ at date $\tau$. In other words, it is the certainty equivalent increase in consumption at date $\tau$ of a unit benefit at date $t$, evaluated from date 0 .

In the following proposition, we link the term structure of efficient discount rates today to the joint distribution of spot one-period-ahead rates and consumption levels. We allow for arbitrary interim evaluation dates $0=\tau_{0}<\tau_{1}<\ldots<\tau_{n}=t$. Although these dates are arbitrary, the idea is to apply Proposition 4 to a partition of $[0, t]$ that is such that a liquid risk free zerocoupon bond can be traded within each subperiod $\left[\tau_{i}, \tau_{i+1}\right]$, so that its equilibrium rate $r_{\tau_{i} \rightarrow \tau_{i+1}}$ and its associated discount factor are economically meaningful.

Proposition 4: There are three equivalent ways to define the efficient long discount rate $r_{0 \rightarrow t}$ :

$$
\text { Ramsey rule: } \quad r_{0 \rightarrow t}=-t^{-1} \ln \frac{E u^{\prime}\left(c_{t}\right)}{u^{\prime}\left(c_{0}\right)}
$$

$$
\text { Expected Future Value: } \quad r_{0 \rightarrow t}=t^{-1} \ln E\left[\frac{u^{\prime}\left(c_{t}\right)}{E u^{\prime}\left(c_{t}\right)} e^{\sum_{i=0}^{n-1}\left(\tau_{i+1}-\tau_{i}\right) r_{\tau_{i} \rightarrow \tau_{i+1}}}\right]
$$

Present Value: $\quad r_{0 \rightarrow t}=t^{-1} \sum_{i=0}^{n-1}\left(\tau_{i+1}-\tau_{i}\right) R_{\tau_{i} \rightarrow \tau_{i+1}}=-t^{-1} \sum_{i=0}^{n-1} \ln E\left[\frac{u^{\prime}\left(c_{\tau}\right)}{E u^{\prime}\left(c_{\tau}\right)} e^{-\left(\tau_{i+1}-\tau_{i}\right) r_{\tau_{i} \rightarrow \tau_{i+1}}}\right]$.

Proof: See the Appendix. 
These three equations can be obtained by examining the impact on social welfare of three different strategies to allocate a sure benefit $F_{t}$ arising at date $t$. The Ramsey rule (18) consists in consuming $F_{t}$ at that date, and to determine which sure increase in consumption today yields the same impact on intertemporal welfare. The "expected future value” rule (19) consists in determining the amount to be invested today in a roll-over investment in the oneperiod-maturity risk free asset that yields an uncertain increase in consumption at date $t$ generating the same increase in welfare as consuming $F_{t}$ at date $t$. Finally, the "present value" rule (20) is obtained by valuing $F_{t}$ through a sequence of backward risk-adjusted PV computations.

None of these rules comes close to the Weitzman's gamma discounting rule $r_{0 \rightarrow t}^{W}$ given by the following equation:

$$
e^{-t r_{0 \rightarrow t}^{W}}=E e^{-\sum_{i=0}^{n-1}\left(\tau_{i+1}-\tau_{i}\right) r_{\tau_{i} \rightarrow \tau_{i+1}}}
$$

As shown in the previous section, for any utility function $u$, there exists an economy $\left.c_{t}\right|_{t \geq 0}$ for which the gamma discounting rule (21) yields inefficient discount rates. Two extreme cases should be considered. Suppose first that $u$ is CRRA and that growth rates $x_{t}$ are i.i.d.. In that case, it is well-known that the term structure of efficient rates is flat, and that interest rates $r_{\tau \rightarrow \tau+1}=-\ln E \exp (-\gamma x)$ are constant. Because future spot rates are certain, the gamma discounting rule is correct, but trivial. The second extreme case arises when assuming that the macroeconomic uncertainty is fully resolved instantaneously right after date $0 .{ }^{10}$ In that case, the gamma discounting rule is efficient, and non-trivial.

Proposition 5: Suppose that the uncertainty affecting growth is fully resolved at date $\tau_{1}$ which shrinks to zero. Then, the gamma discounting rule is efficient: For all $t>0$ : $r_{0 \rightarrow t}^{W}=r_{0 \rightarrow t}$.

Proof: See the Appendix.

More generally, in such an economy, all assets should be valued in a risk-neutral manner! The intuition of this result can be derived in three steps. First, observe that when the uncertainty is immediately resolved at date $\tau_{1}$, the present value $P V_{\tau_{1} \leftarrow t}$ has the coherent economic meaning

\footnotetext{
${ }^{10}$ This case formed the reference model of the first version of this paper.
} 
of the size of a loan at date $\tau_{1}$ to be reimbursed by a sure payment later on. The second step is to recognize that although this present value is uncertain at date 0 , this uncertain benefit comes to increase consumption $c_{\tau_{1}} \approx c_{0}$, which is certain. So, there is no self-insurance benefit for $P V_{\tau_{1} \leftarrow t}$ in this case. Third, remember that risk aversion is a second-order effect in the expected utility model (Segal and Spivak (1990)). ${ }^{11}$ This implies that using the expectation of the present value $P V_{0 \leftarrow t}$ to value a sure benefit at date $t$ is efficient.

The following two examples illustrate the difference between the term structures of the efficient discount rates and of the gamma discount rates. The first example is inspired from Weitzman (1998, 2001, 2010), and from Proposition 5. But contrary to this proposition, we assume that the resolution of the uncertainty is resolved only in 30 years, not today. As in Weitzman (2001, 2010), we calibrate the model in such a way that the future spot interest rate has a gamma distribution.

Example 2: Suppose that $u$ is CRRA with relative risk aversion $\gamma$. We assume that each period lasts 30 years. Suppose also that the uncertainty is fully revealed at date 1 (so, in 30 years), with a constant growth rate forever. This means that, conditional on $x_{1}=x, x_{\tau}$ is equal to $x$ almost surely. This is an extreme form of PQD, yielding a decreasing term structure of efficient discount rates. This implies that the future spot rate $r_{\tau \rightarrow \tau+1}$ conditional on $x_{1}$ is just equal to $\gamma x_{1}$. Let us calibrate this model by assuming that the uncertainty affecting growth rate $x_{1}$ at date 0 is governed by a gamma density function:

$$
f\left(x_{1} ; a, b\right)=x_{1}^{a-1} \frac{e^{-x_{1} / b}}{b^{a} \Gamma(a)} \quad \text { for all } x>0
$$

where $a$ and $b$ are two positive constant. Its mean and variance equal respectively $E x_{1}=\mu=a b$ and $\operatorname{Var}\left(x_{1}\right)=\sigma^{2}=a b^{2}$. We show in the Appendix that

$$
r_{0 \rightarrow t}=\frac{a \ln (1+\gamma b t)}{t}
$$

and

\footnotetext{
${ }^{11}$ This means that, when background consumption is certain, the certainty equivalent of payoff $\varepsilon x$ equals $\varepsilon E x+o\left(\varepsilon^{2}\right)$, with $\lim _{\varepsilon \rightarrow 0} o\left(\varepsilon^{2}\right) / \varepsilon=0$.
} 


$$
r_{0 \rightarrow t}^{W}=\frac{a \ln \left(1+\gamma b t+\gamma^{2} b^{2}(t-1)\right)}{t} .
$$

We calibrate the model with $\gamma=2$ and $\mu=\sigma=1$, so that consumption doubles every thirty years in expectation. This corresponds to $a=b=1$. In Table 1 , we computed the annualized discount rates for different discount rates by using equations (23) and (24).

\begin{tabular}{ccc}
\hline maturity & $\begin{array}{c}\text { Efficient discounting } \\
r_{0 \rightarrow t}\end{array}$ & $\begin{array}{c}\text { Gamma discounting } \\
r_{0 \rightarrow t}^{W}\end{array}$ \\
\hline 30 years $(\mathrm{t}=1)$ & $3.66 \%$ & $3.66 \%$ \\
60 years $(\mathrm{t}=2)$ & $2.68 \%$ & $3.66 \%$ \\
90 years $(\mathrm{t}=3)$ & $2.16 \%$ & $3.01 \%$ \\
120 years $(\mathrm{t}=4)$ & $1.83 \%$ & $2.54 \%$ \\
150 years $(\mathrm{t}=5)$ & $1.60 \%$ & $2.20 \%$ \\
180 years $(\mathrm{t}=6)$ & $1.42 \%$ & $1.94 \%$ \\
\hline \multicolumn{2}{c}{ Table 1: Discount rates for the model presented in Example 2, } \\
\multicolumn{3}{c}{ with $\gamma=2$ and $a=b=1}$.
\end{tabular}

This example confirms the findings of the previous section. Because the shock on future growth rates is permanent, which is a strong form of PQD, the term structure of efficient discount rates is decreasing, and the gamma discounting rule overestimates them. This means that Weitzman' recommendation is short-termist. But this example shares with Gollier and Weitzman (2010) and Weitzman (2010) the unrealistic feature to assume that the uncertainty is fully resolved in the medium term. In our last example, we consider a case in which the uncertainty is smoothly resolved over time.

Example 3: Let us assume as before that the representative agent is CRRA with relative risk aversion $\gamma \geq 0$. We assume that growth rates $x_{\tau}=\ln c_{\tau} / c_{\tau-1}$ per period are i.i.d. normal with mean $\theta$ and variance $\sigma^{2}$. At date 0 , the true value of $\theta$ is uncertain. The beliefs of the representative agent about the mean growth rate are represented by a normal distribution with mean $\mu$ and variance $\sigma_{0}^{2}$, which measures the ambiguity prevailing at that time. Of course, the agent will learn the true value of $\theta$ over time by observing realized growth rates. Technical details are described in the Appendix. Gollier (2008) showed that the efficient discount rates in such an economy are characterized as follows: 


$$
r_{0 \rightarrow t}=\gamma \mu-0.5 \gamma^{2}\left(\sigma^{2}+t \sigma_{0}^{2}\right)
$$

The term structure of efficient discount rates is linearly decreasing. Computing the term structure of the gamma discount rates is more complex. Conditional on the realization of past growth rates $\left(x_{1}, \ldots, x_{\tau}\right)$, the spot interest rate at date $\tau$ is given by

$$
r_{\tau \rightarrow \tau+1} \mid x_{1}, \ldots, x_{\tau}=\gamma \frac{\mu \sigma^{2}+m_{\tau} \tau \sigma_{0}^{2}}{\sigma^{2}+\tau \sigma_{0}^{2}}-0.5 \gamma^{2} \sigma^{2} \frac{\sigma^{2}+(\tau+1) \sigma_{0}^{2}}{\sigma^{2}+\tau \sigma_{0}^{2}}
$$

where $m_{\tau}=\left(x_{1}+\ldots+x_{\tau}\right) / \tau$ is the mean growth rate from 0 to $\tau$. It is shown in the Appendix that the gamma discounting rule yields

$$
r_{0 \rightarrow t}^{W}=\gamma \mu-0.5 \gamma^{2} v_{t}
$$

where $v_{t}$ is defined in the Appendix, with $v_{1}=v_{2}=\sigma^{2}+\sigma_{0}^{2}$. In Figure 1, we draw the term structures of $r_{0 \rightarrow t}$ and of $r_{0 \rightarrow t}^{W}$ in the case of $\gamma=2, \mu=2 \%, \sigma=4 \%, \sigma_{0}=1 \%$. We see again that gamma discounting is short-termist. For example, Weitzman would recommend using $r_{0 \rightarrow 100}^{W}=2.51 \%$ in such an economy, but the efficient discount rate is only $r_{0 \rightarrow 100}=1.68 \%$.

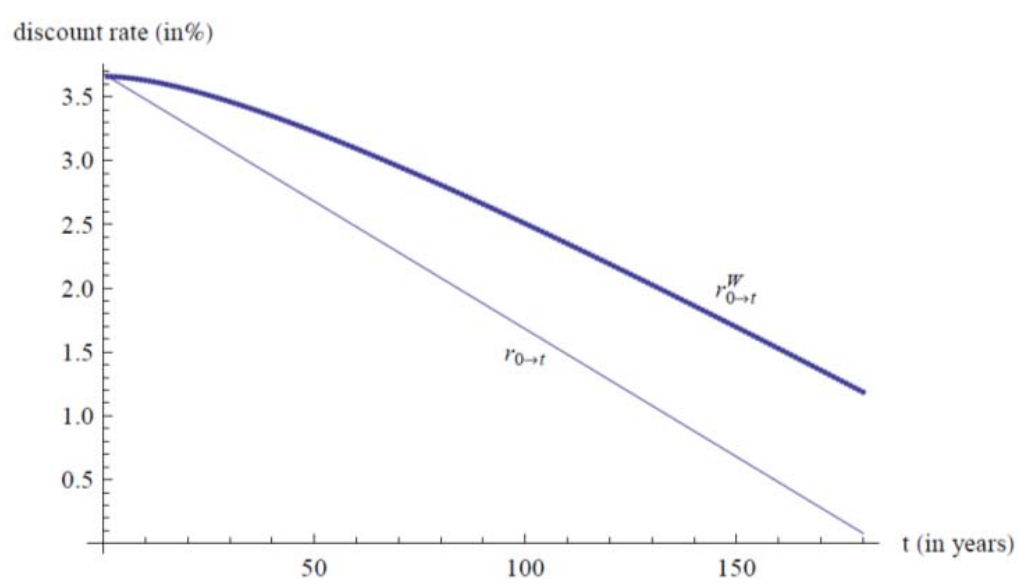

Figure 1: Term structures of $r_{0 \rightarrow t}$ and of $r_{0 \rightarrow t}^{W}$ in with CRRA preferences $(\gamma=2)$ and $x_{1}, x_{2}, \ldots \mid \theta \sim$ i.i.d. $N\left(\theta, \sigma^{2}\right), \theta \sim N\left(\mu, \sigma_{0}^{2}\right)$. We assume that $\mu=2 \%, \sigma=4 \%$, and $\sigma_{0}=1 \%$.

\section{Related literature}

Gollier (2004) was the first to criticize gamma discounting for its weak economic foundation. Gamma discounting is based on the idea that when interest rates are uncertain, one should use 
the expected net present value (NPV) criterion to evaluate safe projects. Gollier showed that the same weak foundation offers an alternative criterion based on the expected net future value (NFV), yielding discount rates $R_{0 \rightarrow t}^{G}$ such that

$$
e^{t R_{0 \rightarrow t}^{G}}=E e^{\sum_{i=0}^{n-1}\left(\tau_{i+1}-\tau_{i}\right) r_{\tau_{i} \rightarrow \tau_{i+1}}}
$$

Under the same beliefs as those considered by Weitzman (1998, 2001), which are similar to example 2 above, it is easy to show that the term structures of $R_{0 \rightarrow t}^{G}$ and $R_{0 \rightarrow t}^{W}$ are respectively increasing and decreasing and they converge asymptotically respectively to the largest and to the smallest possible interest rate. Gollier concluded that "Weitzman and I cannot be both right. In fact, to tell the truth, I believe that we are both wrong, because our criteria are arbitrary”. ${ }^{12}$ This remark has generated an intense debate about which rule should be applied in practice. This is illustrated by the titles of some recent papers on this topic: "Yes, we should discount the far-distant future at its lowest possible rate: A resolution of the Weitzman-Gollier puzzle” (Freeman (2010)); “The Weitzman-Gollier puzzle is not a paradox but a mistake” (Szekeres (2013)); and “Disentangling the Weitzman and the Gollier effect” (Traeger (2013)). This literature did not converge to a consensus in the profession about the efficiency of decreasing term structure of discount rates.

Cox, Ingersoll and Ross (1981) have demonstrated the incompatibility of the different versions of the Expectations Hypothesis with each other and with the standard consumptionbased asset pricing model, except when uncertainty is resolved one period ahead. This means that both the expected NPV and the expected NFV are not sustained by standard finance theory. Pazner and Razin (1975) have been the first to show that the expected NPV and NFV approaches diverge in their recommendation under risk neutrality, but these authors did not attempt to solve the puzzle. Hepburn and Groom (2007) generalized their analysis by showing that one can consider other evaluation dates than 0 (for NPV) or $t$ (for NFV), each choice yielding a different term structure.

These authors do not link interest rate risks to risk on consumption, which is necessary to solve the puzzle. Buchholz and Schumacher (2008) introduced risk aversion into the debate and defined the certainty equivalent discount rate accordingly, but the corresponding decision rule remains sensitive to the date at which the net benefit is consumed. This is because they

\footnotetext{
12 The fact that $r_{0 \rightarrow t}^{G}$ defined by equation (28) is inefficient is obvious from comparing this equation to (19).
} 
did not recognize that the discount rate is linked to the growth of consumption through an equilibrium condition on the credit market.

Freeman (2010) integrated this crucial observation into his analysis. He examined a Lucas tree economy as in this paper, but his analysis is restricted to growth processes with full resolution of uncertainty at date 0 , and with a flat term structure contingent to the signal. Considering the special case of Kreps-Porteus preferences with risk neutrality, he concluded that the expected NPV criterion correctly identifies the attractiveness of social initiatives. However, it is hard to accept the assumption of risk neutrality, in particular in the context of extra-long maturities that is typically considered in this literature on gamma discounting.

Gollier and Weitzman (2010) and Weitzman (2010) showed that the expected NPV and NFV approaches can be reconciled through the addition of risk premia to the NPV and the NFV portfolios. Whereas this paper takes the stochastic growth of consumption as exogenous and derives interest rates from equilibrium conditions, Gollier and Weitzman (2010) and Weitzman (2010) take stochastic interest rates as given. As in our Proposition 5, they also assume unrealistically that the uncertainty about the future is fully resolved right after the investment decision. It implies that they optimally react to the signal at date 0 by adapting their consumption level to changes in their expectation about future interest rates. They showed that gamma discounting and the expected NPV rule is efficient if and only if the representative agent has a logarithmic utility function, which is a striking difference with what we obtain in this paper. The logarithmic case is the only one in which $c_{0}$ is optimally independent of information about future interest rate. In the other cases, $c_{0}$ is not constant, and the second-order risk aversion argument cannot be invoked to get a result as in Proposition 5. Traeger (2013) extended this analysis to the case of risky projects. He showed that measuring the risk-adjusted expected NPV of risky projects combines a "Weitzman effect" and a "Gollier effect" coming respectively from the correlated market interest rates and from the correlated rates of return of the project under scrutiny.

It is useful to link this literature to the more traditional asset pricing theory. This link comes from rule (18), which is the classical CCAPM risk free asset pricing rule. Gollier (2012) showed that this equation generates a decreasing term structure of discount rates when future spot growth rates of consumption are PQD, and he illustrated this result with different stochastic growth processes exhibiting some persistence of shocks. 
Newell and Pizer (2003), Groom, Koundouri, Panopoulou and Pantelidis (2007), and Gollier, Koundouri and Pantelidis (2008) have calibrated the expected net present value rule by using time series data on interest rates. They estimated various stochastic processes, many of them yielding a sizeable degree of persistence in the dynamics of these rates, and therefore a strongly decreasing term structure of gamma discounting rates $r_{0 \rightarrow t}^{W}$. Farmer, Geanakoplos, Masoliver, Montero and Perello (2014) fit the gamma discounting formula with a OrnsteinUhlenbeck (mean-reversion) process for spot rates by using historical data across 14 countries covering 87 to 318 years. They show that the rate at which one should discount very distant safe benefits can be substantially less than the historical mean spot interest rate. Cropper, Freeman, Groom and Pizer (2014) provide a recent short survey of this literature.

Gamma discounting is remotely related to the fundamental theorem of finance (Rubinstein (1976), Ross (1978)) which states that any no-arbitrage pricing rule can be represented by a price kernel (or stochastic discount factor) $m_{t}$ such that any asset with a payoff $F_{t}$ occurring at date $t$ has a price equaling $E m_{t} F_{t}$. If $F_{t}$ is certain, and if we use a representation $m_{t}=\exp (-r t)$ for the price kernel, then we formally have that the value of the safe asset is equal to $F_{t}$ discounted at rate $r_{0 \rightarrow t}^{W}$. But this potential justification of gamma discounting is mostly semantic, for two reasons. First, this approach requires that the price kernel be an exponential function of time, with a "stochastic discount rate" $r$ being independent of time. Second, and more importantly, it is misleading to identify $r$ as an "interest rate", as done for example in Weitzman (2001) $)^{13}$ and in subsequent works aimed at estimating gamma discounting rates from data on interest rates. Such an identification would be correct only if the price $m_{t}(s)$ of the Arrow-Debreu security that delivers one dollar at date $t$ in growth scenario $s$ would be equal to the present value of that dollar discounted at the compounded interest rate observed in that scenario. As seen in this paper, this hypothesis is false when the uncertainty is resolved only gradually.

We do not cover here the literature associated with alternative interpretations of the source of uncertainty affecting future interest rates. In Jouini, Marin and Napp (2010) and Freeman and Groom (2012), economic agents agree to disagree about their expectations relative to consumption growth. In Gollier and Zeckhauser (2005) and Heal and Millner (2013),

\footnotetext{
${ }^{13}$ Weitzman (2001) calibrated the uncertainty on $r$ by the following survey question: "What real interest rate do you think should be used to discount over time the (expected) benefits and (expected) costs of projects being proposed to mitigate the possible effects of global climate change?”
} 
consumers have heterogeneous time preferences. Under some conditions, the term structure of the rate of impatience of the representative consumer is decreasing with time, as in gamma discounting. In both cases, the problem examined by Weitzman $(1998,2001)$ is interpreted as an aggregation problem of beliefs or preferences.

\section{Conclusion}

Following Weitzman (1998, 2001), several countries have decided -- or are considering the possibility - to implement gamma discounting to evaluate public policies. But we have shown here that, when future interest rates are uncertain, there is no social preference that generically supports gamma discounting and its underlying criterion, the expected net present value rule. The beauty of gamma discounting is that the term structure of discount rates can be derived from the sole distribution of future interest rates. Using a standard Lucas tree economy, we have shown that it is not possible to characterize the term structure of efficient discount rates with just that information. The distribution of future interest rates is not a sufficient statistic for recovering the term structure of efficient discount rates today.

If future interest rates are uncertain, transferring future benefit through time -- either backward to compute the NPV, or forward to compute the NFV -- yields risk. Depending upon the correlation between the return of this financial operation and the growth rate of aggregate consumption, the evaluation of the project requires taking into account of a risk premium, which can be positive or negative. Under gamma discounting, one implicitly assumes that this risk premium is always zero, thereby making a mistake that is as large as if one would assume that any risky asset should be evaluated in a risk neutral manner.

We have shown that this risk premium is negative when shocks on consumption growth are persistent. The intuition is that future consumption is positively correlated with future spot interest rates in that case, which implies that future short-term bonds have a negative CCAPM beta. The risk neutrality assumption implicit in gamma discounting tends to undervalue sure future benefits by ignoring the hedging benefit of the PV strategy. Thus gamma discounting is short termist, yielding discount rates that are too large. The bottom line of this paper is that gamma discounting is not supported by standard economic principles.

However, Weitzman (2001) is mostly right, but for the wrong reasons. Indeed, we have shown in this paper that there are good reasons to recommend using a decreasing term 
structure of discount rates. Bansal and Yaron (2004) and many others have shown that shocks to the growth rate of consumption are persistent. This tends to magnify the long run risk faced by future generations. Under prudence, this should induce us to invest more in projects generating safe benefits in the distant future. This should be done by using discount rates with a decreasing term structure. 


\section{References}

Arrow, K., M. Cropper, C. Gollier, B. Groom, G. Heal, R. Newell, W. Nordhaus, R. Pindyck, W. Pizer, P. Portney, T. Sterner, R. Tol, and M. Weitzman, (2013), Determining benefits and costs for future generations, Science, 341, 349-350.

Arrow, K., M. Cropper, C. Gollier, B. Groom, G. Heal, R. Newell, W. Nordhaus, R. Pindyck, W. Pizer, P. Portney, T. Sterner, R. Tol, and M. Weitzman, (2014), Should a Declining Discount Rate Be Used in Project Analysis?, Review of Environmental Economics and Policy, 8, 145-163.

Arrow, K.J., and R.C. Lind, (1970), Uncertainty and the evaluation of public investment decision, American Economic Review, 60, 364-378.

Bansal, R., and A. Yaron, (2004), Risks For the Long Run: A Potential Resolution of Asset Pricing Puzzles, Journal of Finance 59, 1481-1509.

Buchholz, W., and J. Schumacher, (2008), Discounting the long distant future: Simple explanation for the Weitzman-Gollier-puzzle, mimeo.

Cox, J.C., J.E. Ingersoll, and S. Ross, (1981), A reexamination of traditional hypotheses about the term structure of interest rates, Journal of Finance 36, 769-799.

Crooper, M.L., M.C. Freeman, Groom B., and W.A. Pizer, (2014), Declining discount rates, American Economic Review Papers and Proceeding, 104(5), 538-543.

Farmer, J.D., J. Geanakoplos, J. Masoliver, M. Montero, and J. Perello, (2014), Discounting the distant future, Cowles Foundation Discussion Paper n 1951, New Haven.

Freeman, M.C., (2010), Yes, we should discount the far-distant future at its lowest possible rate: A resolution of the Weitzman-Gollier puzzle, The Open-Access, Open-Assessment EJournal 4.

Freeman, M.C., and B. Groom, (2012), Gamma discounting the Combination of forecasts, unpublished paper.

Froot, K.A., (1989), New hope for the expectations hypothesis of the term structure of interest rates, Journal of Finance 44, 283-305. 
Gollier, C., (2004), Maximizing the expected net future value as an alternative strategy to gamma discounting." Finance Research Letters 1 (2): 85-89.

Gollier, C., (2008), Discounting with fat-tailed economic growth, Journal of Risk and Uncertainty, 37, 171-186.

Gollier, C., (2012), Pricing the planet's future: The economics of discounting in an uncertain world, Princeton University Press, Princeton.

Gollier, C., (2014), Discounting and growth, American Economic Review Papers and Proceedings, 104 (5), 534-537.

Gollier, C., P. Koundouri, and T. Pantelidis, (2008), Declining discount rates: Economic justifications and implications for long-run policy, Economic Policy (56), 757-795.

Gollier, C., and M.L. Weitzman, (2010), How Should the Distant Future be Discounted When Discount Rates are Uncertain?, Economic Letters, 107(3), 350-353.

Gollier, C., and R.H. Zeckhauser, (2005), Aggregation of heterogeneous time preferences, Journal of Political Economy 113, 878-896.

Gilles, C., and S.F. Leroy, (1986), A note on the local expectations hypothesis: A discretetime exposition, Journal of Finance 41, 975-979.

Groom, B., P. Koundouri, E. Panopoulou and T. Pantelidis, (2007), Discounting the distant future: how much does model selection affect the certainty equivalent rate?, Journal of Applied Econometrics, 22 (3), 641-656.

Heal, G., and A. Millner, (2013), Discounting under disagreement, unpublished manuscript.

Hepburn, C., and B. Groom, (2007), Gamma discounting and expected net future value, Journal of Environmental Economics and Management (53), 99-109.

HM Treasury, (2003), The Green Book - Appraisal and evaluation in central government. London.

Jouini, E., J.-M. Marin, and C. Napp, (2010), Discounting and divergence of opinion, Journal of Economic Theory 145, 830-859. 
Kimball, M.S., (1990), Precautionary savings in the small and in the large, Econometrica, 58, 53-73.

Lebègue, D, (2005), Révision du taux d'actualisation des investissements publics, Commissariat Général au Plan, Paris.

Lehmann, E., (1966), Some concepts of dependence, Annals of Mathematical Statistics, 37, 1137-1153.

Levy, H., and J. Paroush, (1974), Toward multivariate efficiency criteria, Journal of Economic Theory 7, 129-42.

Lucas, R., (1978), Asset prices in an exchange economy, Econometrica, 46, 1429-46.

Macaulay, F.R., (1938), Some theoretical problems suggested by the movements of interest rates, bonds yields, and stocks prices in the United States since 1856, NBER Working Papers Series, New York.

Meyer, M., and B. Strulovici, (2012), Increasing interdependence in multivariate distributions, Journal of Economic Theory 147, 1460-1489.

Newell, R. and W. Pizer, (2003), Discounting the benefits of climate change mitigation: How much do uncertain rates increase valuations? Journal of Environmental Economics and Management, 46 (1), 52-71.

Official Norwegian Report, (2012), Cost-benefit analysis, NOU 2012:16, Oslo.

Pazner, E. A. and A. Razin, (1975), On expected present value vs. expected future value, Journal of Finance 30 (3): 875-877.

Pearce, D., G. Atkinson and S. Mourato, (2006), Cost-benefit analysis and the environment: Recent developments, Paris: OECD.

Piazzesi, M., (2010), Affine Term Structure Models, Handbook of Financial Econometrics Volume 1, Chapter 12, pp. 691-766, edited by Y. Ait-Sahalia and L.P. Hansen, North Holland, Elsevier

Ross, S.A., (1978), A simple approach to the valuation of risky streams, Journal of Business, 51 (3), 453-475. 
Rubinstein, M., (1976), The valuation of uncertain income streams and the pricing of options, The Bell Journal of Economics 7 (2), 407-425.

Segal, U. and A. Spivak, (1990), First order versus second order risk aversion, Journal of Economic Theory, 51, 111-125.

Szekeres, S., (2013), The "Weitzman-Gollier puzzle" is not a paradox but a mistake, and it is most likely moot, Open Science Repository Economics, DOI 10.7392/openaccess.23050448.

Traeger, C., (2013), Discounting under uncertainty: Disentangling the Weitzman and the Gollier effect, Journal of Environmental Economics and Management, forthcoming.

Weitzman, M.L., (1998), Why the far-distant future should be discounted at its lowest possible rate?, Journal of Environmental Economics and Management, 36, 201-208.

Weitzman, M.L., (2001), Gamma discounting, American Economic Review, 91, 260-271.

Weitzman, M.L., (2010), Risk-adjusted gamma discounting, Journal of Environmental Economics and Management, 60, 1-13. 


\section{Appendix for Online Publication}

\section{Technical details for Example 1}

We use the following well-known Lemma.

Lemma 1: If $y \sim N\left(\mu_{y}, \sigma_{y}^{2}\right)$, then $E \exp (A y)=\exp \left(A \mu_{y}+0.5 A^{2} \sigma_{y}^{2}\right)$.

We normalize $c_{0}=1$, so that $\ln c_{1} \sim N\left(\mu, \sigma^{2}\right), \ln c_{2} \sim N\left(2 \mu, 2 \sigma^{2}(1+\rho)\right)$ and $\ln \left(c_{2} / c_{1}\right) \mid c_{1}$ is normal with mean $(1-\rho) \mu+\rho \ln c_{1}$ and variance $\left(1-\rho^{2}\right) \sigma^{2}$. This implies from equations (2) and (3) that

$$
\begin{gathered}
e^{-r_{0 \rightarrow 1}}=E e^{-\gamma \ln c_{1}}=e^{-\gamma \mu+0.5 \gamma^{2} \sigma^{2}} \\
e^{-2 r_{0 \rightarrow 2}}=E e^{-\gamma \ln c_{2}}=e^{-2 \gamma \mu+\gamma^{2} \sigma^{2}(1+\rho)}
\end{gathered}
$$

and

$$
e^{-r_{1 \rightarrow 2}}=E e^{-\gamma \ln c_{2} / c_{1}}=e^{-\gamma(1-\rho) \mu-\gamma \rho \ln c_{1}+0.5 \gamma^{2} \sigma^{2}\left(1-\rho^{2}\right)} .
$$

This implies that

$$
\begin{aligned}
e^{-2 r_{0 \rightarrow 2}^{W}} & =E e^{-r_{0 \rightarrow 1}-r_{1 \rightarrow 2}}=e^{-\gamma(2-\rho) \mu+0.5 \gamma^{2} \sigma^{2}\left(2-\rho^{2}\right)} E e^{-\gamma \rho \ln c_{1}} \\
& =e^{-\gamma(2-\rho) \mu+0.5 \gamma^{2} \sigma^{2}\left(2-\rho^{2}\right)} e^{-\gamma \rho \mu+0.5 \gamma^{2} \rho^{2} \sigma^{2}} \\
& =e^{-2 \gamma \mu+\gamma^{2} \sigma^{2}} .
\end{aligned}
$$

\section{Proof of Proposition 4}

Equation (18) is a special case of (16) when $\tau=0$. We now show that equations (18) and (19) are equivalent. By the law of iterated expectations, we have that equation (19) can be rewritten as follows: 


$$
\begin{aligned}
e^{t r_{0 \rightarrow t}} & =E\left[\frac{u^{\prime}\left(c_{t}\right)}{E u^{\prime}\left(c_{t}\right)} e^{\sum_{i=0}^{n-1} r_{\tau_{i} \rightarrow \tau_{i+1}}}\right] \\
& =E E_{\tau_{n-1}}\left[\frac{u^{\prime}\left(c_{0}\right)}{E u^{\prime}\left(c_{\tau_{1}}\right)} \frac{u^{\prime}\left(c_{\tau_{1}}\right)}{E_{\tau_{1}} u^{\prime}\left(c_{\tau_{2}}\right)} \ldots \frac{u^{\prime}\left(c_{\tau_{n-1}}\right)}{E_{\tau_{n-1}} u^{\prime}\left(c_{t}\right)} \frac{u^{\prime}\left(c_{t}\right)}{E u^{\prime}\left(c_{t}\right)}\right] \\
& =E\left[\frac{u^{\prime}\left(c_{0}\right)}{E u^{\prime}\left(c_{\tau_{1}}\right)} \frac{u^{\prime}\left(c_{\tau_{1}}\right)}{E_{\tau_{1}} u^{\prime}\left(c_{\tau_{2}}\right)} \ldots \frac{u^{\prime}\left(c_{\tau_{n-2}}\right)}{E_{\tau_{n-2}} u^{\prime}\left(c_{\tau_{n-1}}\right)} E_{\tau_{n-1}}\left[\frac{u^{\prime}\left(c_{\tau_{n-1}}\right)}{E_{\tau_{n-1}} u^{\prime}\left(c_{t}\right)} \frac{u^{\prime}\left(c_{t}\right)}{E u^{\prime}\left(c_{t}\right)}\right]\right] \\
& =E\left[\frac{u^{\prime}\left(c_{0}\right)}{E u^{\prime}\left(c_{\tau_{1}}\right)} \frac{u^{\prime}\left(c_{\tau_{1}}\right)}{E_{\tau_{1}} u^{\prime}\left(c_{\tau_{2}}\right)} \ldots \frac{u^{\prime}\left(c_{\tau_{n-2}}\right)}{E_{\tau_{n-2}} u^{\prime}\left(c_{\tau_{n-1}}\right)} \frac{u^{\prime}\left(c_{\tau_{n-1}}\right)}{E u^{\prime}\left(c_{t}\right)}\right] .
\end{aligned}
$$

Taking again the expectation conditional on information at date $t-1$ yields in the same way

$$
e^{t r_{0 \rightarrow t}}=E\left[\frac{u^{\prime}\left(c_{0}\right)}{E u^{\prime}\left(c_{\tau_{1}}\right)} \frac{u^{\prime}\left(c_{\tau_{1}}\right)}{E_{\tau_{1}} u^{\prime}\left(c_{\tau_{2}}\right)} \ldots \frac{u^{\prime}\left(c_{\tau_{n-2}}\right)}{E u^{\prime}\left(c_{t}\right)}\right]
$$

Proceeding sequentially in the same backward way, we eventually obtain

$$
e^{t r_{0 \rightarrow t}}=\frac{u^{\prime}\left(c_{0}\right)}{E u^{\prime}\left(c_{t}\right)}
$$

which is equivalent to equation (18). Finally, we show that equation (20) is also equivalent to this equation. Indeed, by definition (17) of future rates $R_{\tau \rightarrow t}$, equation (20) can be rewritten as

$$
e^{-t r_{0 \rightarrow t}}=e^{-\sum_{i=0}^{n-1}\left(\tau_{i+1}-\tau_{i}\right) R_{\tau_{i} \rightarrow \tau_{i+1}}}=\prod_{i=0}^{n-1} \frac{E u^{\prime}\left(c_{\tau_{i+1}}\right)}{E u^{\prime}\left(c_{\tau_{i}}\right)}=\frac{E u^{\prime}\left(c_{t}\right)}{u^{\prime}\left(c_{0}\right)},
$$

which is equation (18).

\section{Proof of Proposition 5}

Without loss of generality, let us decompose $[0, t]$ into two subperiods $\left[0, \tau_{1}\right]$ and $\left[\tau_{1}, t\right]$. Let $s$ denote the signal obtained at $\tau_{1}$. Conditional on $s$, the consumption path $\left.c_{t}\right|_{t \geq 0}$ is deterministic, and denoted $\left.c_{t s}\right|_{t \geq 0}$. We have that $r_{0 \rightarrow \tau_{1}}=-\tau_{1}^{-1} \ln E u^{\prime}\left(c_{\tau_{1}}\right) / u^{\prime}\left(c_{0}\right)$. Because the uncertainty is fully resolved at date $\tau_{1}$, we also have that $r_{\tau_{1} \rightarrow t}(s)=-\left(t-\tau_{1}\right)^{-1} \ln u^{\prime}\left(c_{t s}\right) / u^{\prime}\left(c_{\tau_{1} s}\right)$. It implies that 


$$
\begin{aligned}
e^{-t r_{0 \rightarrow t}^{W}} & =e^{-\tau_{1} r_{0 \rightarrow \tau_{1}}} E e^{-\left(t-\tau_{1}\right) r_{\tau_{1} \rightarrow t}} \\
& =\frac{E u^{\prime}\left(c_{\tau_{1}}\right)}{u^{\prime}\left(c_{0}\right)} E \frac{u^{\prime}\left(c_{t}\right)}{u^{\prime}\left(c_{\tau_{1}}\right)} .
\end{aligned}
$$

When $\tau_{1}$ tends to zero, $c_{\tau_{1}}$ tends to $c_{0}$ with probability 1 , and the above equation can be rewritten as

$$
e^{-t r_{0 \rightarrow t}^{W}}=\frac{E u^{\prime}\left(c_{t}\right)}{u^{\prime}\left(c_{0}\right)}
$$

From equation (18), this implies that $r_{0 \rightarrow t}^{W}=r_{0 \rightarrow t}$. $\square$

\section{Technical details for Example 2}

From equation (18), we have that ${ }^{14}$

$$
e^{-r_{0 \rightarrow t}}=E e^{-\gamma t x_{1}}=\left(\int_{0}^{\infty} x^{a-1} \frac{e^{-\left(b^{-1}+\gamma t\right) x}}{b^{a} \Gamma(a)} d x\right)=(1+\gamma b t)^{-a}
$$

This is equivalent to equation (23). Now observe that because $r=\gamma x$, the future spot rate also has a gamma distribution with parameters $a$ and $\gamma b$. The gamma discounting approach yields

$$
e^{-t r_{0 \rightarrow t}^{W}}=e^{-r_{0 \rightarrow 1}} E e^{-\gamma(t-1) x_{1}}=e^{-a \ln (1+\gamma b)} \int_{0}^{\infty} x^{a-1} \frac{e^{-\left(b^{-1}+\gamma(t-1)\right) x}}{b^{a} \Gamma(a)} d x=(1+\gamma b)^{-a}(1+\gamma b(t-1))^{-a} .
$$

This is equivalent to equation (24).

\section{Technical details for Example 3}

In this example, we have that

$$
\begin{aligned}
& x_{1}, x_{2}, \ldots \mid \theta \sim \text { i.i.d. } N\left(\theta, \sigma^{2}\right) \\
& \theta \sim N\left(\mu, \sigma_{0}^{2}\right)
\end{aligned}
$$

\footnotetext{
${ }^{14}$ We use the property that $\int_{0}^{\infty} x^{h-1} e^{-x / k} d x=k^{h} \Gamma(h)$ for all $k, h>0$.
} 
Under $u^{\prime}(c)=c^{-\gamma}$, Lemma 1 implies that

$$
\begin{aligned}
r_{0 \rightarrow t} & =-t^{-1} \ln E\left[E\left[e^{-\gamma \sum_{t=1}^{t} x_{\tau}} \mid \theta\right]\right]=-t^{-1} \ln E e^{-\gamma t \theta+0.5 \gamma^{2} t \sigma^{2}} \\
& =-0.5 \gamma^{2} \sigma^{2}-t^{-1} \ln e^{-\gamma t \mu+0.5 \gamma^{2} t^{2} \sigma_{0}^{2}} \\
& =\gamma \mu-0.5 \gamma^{2}\left(\sigma^{2}+t \sigma_{0}^{2}\right) .
\end{aligned}
$$

We also know that this implies that

$$
x_{t} \sim N\left(\mu, \sigma^{2}+\sigma_{0}^{2}\right)
$$

and

$$
x_{t+1} \mid x_{1}, \ldots, x_{t} \sim N\left(\frac{\mu \sigma^{2}+m_{t} t \sigma_{0}^{2}}{\sigma^{2}+t \sigma_{0}^{2}}, \sigma^{2} \frac{\sigma^{2}+(t+1) \sigma_{0}^{2}}{\sigma^{2}+t \sigma_{0}^{2}}\right),
$$

Lemma 1 implies that

$$
r_{\tau \rightarrow \tau+1} \mid x_{1}, \ldots, x_{\tau}=\gamma \frac{\mu \sigma^{2}+m_{\tau} \tau \sigma_{0}^{2}}{\sigma^{2}+\tau \sigma_{0}^{2}}-0.5 \gamma^{2} \sigma^{2} \frac{\sigma^{2}+(\tau+1) \sigma_{0}^{2}}{\sigma^{2}+\tau \sigma_{0}^{2}},
$$

with $m_{0}=0$ and $m_{\tau}=\left(x_{1}+\ldots+x_{\tau}\right) / \tau$. We have that

$$
\begin{aligned}
r_{0 \rightarrow t}^{W} & =-t^{-1} \ln E e^{-\sum_{\tau=0}^{t-1} r_{\tau \rightarrow \tau+1}} \\
& =-0.5 \gamma^{2} \sigma^{2} h_{t} t^{-1}-t^{-1} \ln E \exp \left(-\sum_{\tau=0}^{t-1} \gamma \frac{\mu \sigma^{2}+m_{\tau} \tau \sigma_{0}^{2}}{\sigma^{2}+\tau \sigma_{0}^{2}}\right) .
\end{aligned}
$$

with $h_{t}=\sum_{\tau=0}^{t-1}\left(\sigma^{2}+(\tau+1) \sigma_{0}^{2}\right)\left(\sigma^{2}+\tau \sigma_{0}^{2}\right)^{-1}$. Conditional on $\theta$, the expression in the exponential is normally distributed with mean $M_{t}=-\gamma \sum_{\tau=0}^{t-1}\left(\mu \sigma^{2}+\theta \tau \sigma_{0}^{2}\right)\left(\sigma^{2}+\tau \sigma_{0}^{2}\right)^{-1}$ and variance $\gamma^{2} \sigma^{2} \sigma_{0}^{4} k_{t}$, with $k_{t}=\sum_{\tau=1}^{t-1}\left(\sum_{i=\tau}^{t-1}\left(\sigma^{2}+i \sigma_{0}^{2}\right)^{-1}\right)^{2}$. This implies that the above equation can be rewritten as

$$
r_{0 \rightarrow t}^{W}=-0.5 \gamma^{2} \sigma^{2} h_{t} t^{-1}-0.5 \gamma^{2} \sigma^{2} \sigma_{0}^{4} k_{t} t^{-1}-t^{-1} \ln E e^{-\gamma \sum_{\tau=0}^{t-1}\left(\mu \sigma^{2}+\theta \tau \sigma_{0}^{2}\right)\left(\sigma^{2}+\tau \sigma_{0}^{2}\right)^{-1}}
$$


Now, observe that $M_{t}$ is normal with mean $-\gamma \mu t$ and variance $\gamma^{2} \sigma_{0}^{2} l_{t}$ with $\sqrt{l_{t}}=\sum_{\tau=0}^{t-1} \tau \sigma_{0}^{2}\left(\sigma^{2}+\tau \sigma_{0}^{2}\right)^{-1}$. Equation (47) is thus equivalent to

$$
r_{0 \rightarrow t}^{W}=\gamma \mu-0.5 \gamma^{2} v_{t}
$$

with

$$
v_{t}=t^{-1}\left(\sigma^{2} h_{t}+\sigma^{2} \sigma_{0}^{4} k_{t}+\sigma_{0}^{2} l_{t}\right)
$$

